(and one of them is an author on four of the 21 papers I saw); half of the first authors hail from Germany, too, and most of the rest are from Western Europe. If the journal wishes to have an international impact, it will surely need to broaden its author base.

There are certainly good reasons why authors might want to consider this journal. There are no page charges, for a start. Despite this, production values are pretty high: the layout is easy on the eye, and the figures are crisp and clear (although colour figures are only published electronically). Authors also receive 25 free offprints. Publication time seems fairly fast - about five months from submission on average - although this will presumably rise as resubmissions work their way through the system. I can't see many individuals wanting to buy the journal, however: it covers too wide a range of taxa (so far) and too narrow a range of questions, and is anyway priced more for institutions.

If it can keep its publication time down, keep its production values high and broaden its geographic pull, then Organisms, Diversity and Evolution has every chance of establishing itself as a valued library journal. It will be interesting to see whether it begins to narrow its focus, as many previous journals have, or maintains its diversity of subject matter and approach.

Andy Purvis is in the Department of Biological

Sciences, Imperial College, Silwood Park,

Ascot SL5 7PY, UK.

www.urbanfischer.de/journals/ode/organism.htm

\section{In the land}

\section{of the giants}

\section{Comparative and Functional \\ Genomics \\ editor-in-chief Steve Oliver \\ Wiley. 6/yr. \$240 (institutional); \\ $\$ 180$ (individual)}

\section{Greg Elgar}

Less than a decade ago, some expression data, a few kilobases of sequence and an alignment were still sufficient to wring a publication out of Science, Nature and other high-brow publications. Nowadays, of course, scientists need to come up with whole genomes. Such is the pace of progress that even publication in more specialized journals such as Genomics and Genome Research requires mega- or even tera-buckets of data, beyond the scope of the average lab. For those researchers (most of us actually) who work in the smaller lab environment, it is vital that public databases are mined and interpreted to their full extent, both to enrich our knowledge of specific genes or pathways and to allow the design of more effective experiments. It is equally import-

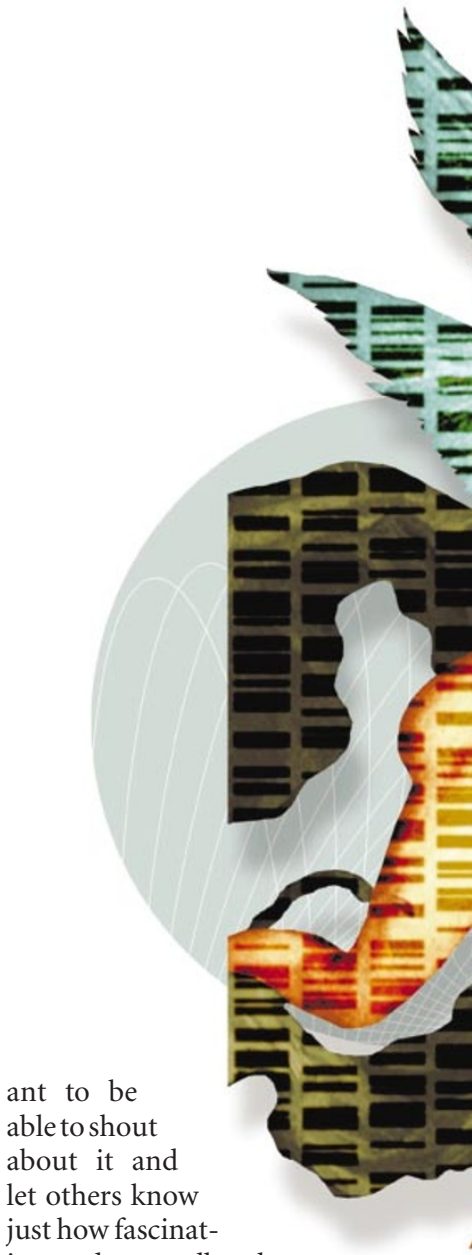

just how fascinat-

ing and groundbreak-

ing your research really is.

The use of comparative genomics as a means to investigate gene function is a direct result of the wealth of genomic data in public databases, and has led to its widespread application in dissecting gene function. It is therefore essential that this new discipline has a dedicated journal in which to disseminate these findings. Comparative and Functional Genomics fills the void and is appropriately forward thinking and looking in concept and design. As well as presenting a (currently slightly thin) selection of original articles and reviews, it includes comprehensive coverage of meetings in the field, as well as a particularly useful 'Current awareness' section, which lists recently published reviews and papers by subject matter.

Despite the organization and clarity of many large public databases, a huge amount of data and tools lurk in an enormous number of 'genomics' websites - well over halfa-million pages according to a search on Google (http://www.google.com). Perhaps in recognition of this Internet genomics jungle (or perhaps jumble is more appropriate), Comparative and Functional Genomics regularly carries a website review, which usually involves a jolly good trawl through some of the myriad sites surrounding a particular topic, such as cancer.

The quality of the reviews and original research published in the journal is high, 
Universe. It is an interdisciplinary subject that has only fairly recently attained scientific respectability. Gathered under the banner of astrobiology is a rich assortment of scientists, including astronomers, biologists, chemists, geologists, microbiologists, palaeontologists and planetary scientists, and astrobiology provides a place for them to exchange ideas and information. The recognition of this discipline has probably changed neither the subject matter of the research carried out, nor altered the way in which the research is done, but perhaps the label of 'astrobiologist' has helped to shift the emphasis of particular projects.

The diverse subject matter of astrobiology has resulted in the publication of papers across a variety of journals, and - efficient online search engines notwithstanding unfamiliarity with a subject could result in contributions being overlooked. As a corollary, papers that have a cross-disciplinary focus might not find a comfortable home in journals that are becoming increasingly specialist. It is therefore extremely timely that the discipline should spawn its own journals.

Astrobiology is aimed at professional scientists but, because of the nature of the subject, the papers are more general than are usually found in the scientific literature. This does not detract from the journal indeed, it could be an extremely valuable aspect that is worth developing by the publication of review papers aimed at informing the community.

The journal was launched in spring 2001, and is beautifully produced, with an eye-catching cover. It seems to have successfully attracted both the microbial- and planetary-science communities, with papers covering, among others, the silicification of bacteria, the chemistry of adenine, the evolution of photosynthetic organisms on Earth, and the exploration of Mars and Europa. Eventually, it is to be anticipated, there will be contributions with a more astronomicalbias.

The journal offers 'Astrobiology Literaturewatch', a documentation of papers published on astrobiology. The list, which runs to 26 pages in the winter 2001 printed issue of the journal, is certainly thorough, and provides a useful summary of the current state of the literature, but it is much more useful as an online resource. As is now expected, Astrobiology is also available electronically.

In this rapidly growing and broad field, it is entirely appropriate that a new journal should emerge. It will be interesting to see how, like life itself, it evolves, adapts and survives.

Monica M. Grady is in the Department of Mineralogy, The Natural History Museum, Cromwell Road, London SW7 5BD, UK. She is an associate editor of the International Journal of Astrobiology, launched only this year by Cambridge University Press and so ineligible for review in this year's round-up of new journals. www.liebertpub.com/ast

\section{Noise worth listening to}

\section{Fluctuation and Noise Letters editor-in-chief L. B. Kish \\ World Scientific. 4/yr. \$280 (institutional); $\$ 112$ (individuals, print only)}

\section{Katja Lindenberg}

A surge of interest in systems on the small scale is to be found everywhere in the scientific and popular literature. This interest arises from the context: many of us find biological systems and devices on the nanoscale to be intriguing or fascinating. The enormous progress in the understanding of the nanoscale world has been fuelled by developments in fields such as nanotech-
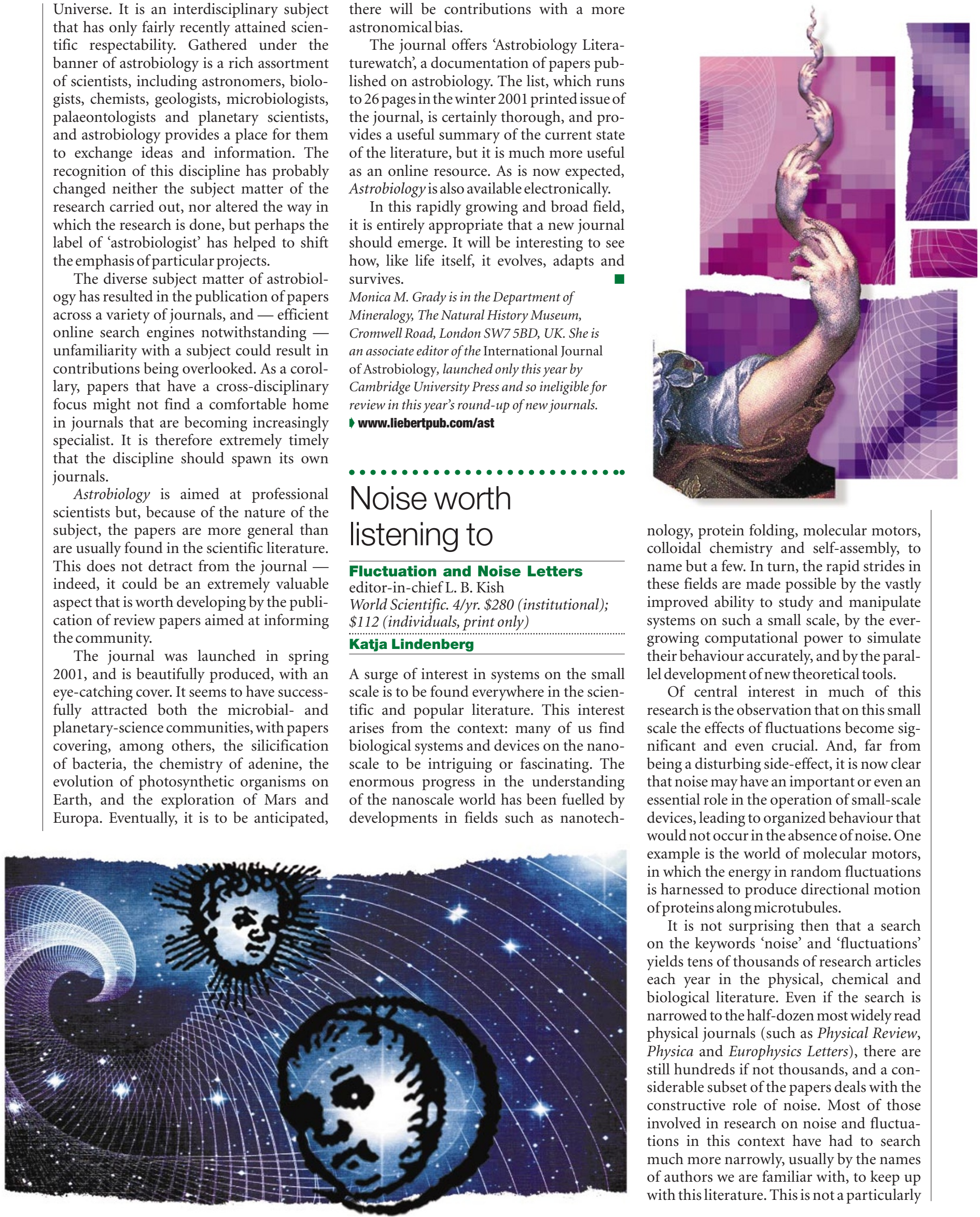

nology, protein folding, molecular motors, colloidal chemistry and self-assembly, to name but a few. In turn, the rapid strides in these fields are made possible by the vastly improved ability to study and manipulate systems on such a small scale, by the evergrowing computational power to simulate their behaviour accurately, and by the parallel development of new theoretical tools.

Of central interest in much of this research is the observation that on this small scale the effects of fluctuations become significant and even crucial. And, far from being a disturbing side-effect, it is now clear that noise may have an important or even an essential role in the operation of small-scale devices, leading to organized behaviour that would not occur in the absence of noise. One example is the world of molecular motors, in which the energy in random fluctuations is harnessed to produce directional motion of proteins along microtubules.

It is not surprising then that a search on the keywords 'noise' and 'fluctuations' yields tens of thousands of research articles each year in the physical, chemical and biological literature. Even if the search is narrowed to the half-dozen most widely read physical journals (such as Physical Review, Physica and Europhysics Letters), there are still hundreds if not thousands, and a considerable subset of the papers deals with the constructive role of noise. Most of those involved in research on noise and fluctuations in this context have had to search much more narrowly, usually by the names of authors we are familiar with, to keep up with this literature. This is not a particularly 